\title{
Le niveau d'expression du gène de l'insuline est-il relié à la susceptibilité au diabète de type 1 ?
}

Les études génétiques ont montré qu'existaient de nombreux gènes de susceptibilité au diabète de type 1, c'est-à-dire à la forme insulinodépendante liée à une destruction autoimmune des cellules $\beta$ des îlots de Langerhans. Le locus IDDM1 réside dans la région du chromosome 6 où siègent les gènes du complexe majeur d'histocompatibilité [1]. Le locus IDDM2 a, quant à lui, été localisé à un fragment de $4,1 \mathrm{~kb}$ contenant le gène de l'insuline ainsi que les régions bordantes en 3' et en 5'. Le motif candidat le plus probable est une séquence répétée VNTR (variable number of tandem repeats) dont l'élément dè base est formé d'une séquence de $14 \mathrm{pb}$. Ce VNTR est extrêmement polymorphe et il en existe au moins une douzaine de formes différentes que l'on peut ranger en trois classes: la classe I, courte, composée d'environ 40 répétitions; la classe II, intermédiaire, composée d'environ 85 répétitions; et enfin la classe III, longue, possédant environ 150 répétitions. C'est l'homozygotie pour des allèles de classe I qui semble augmenter la susceptibilité au diabète [2]. Le VNTR se terminant 3654 paires de bases en amont du site de début de la transcription, la question de son effet sur le niveau de transcription méritait d'être posée. En fait, les premiers résultats disponibles sont contradictoires. Kennedy et al. (San Francisco, CA et Cambridge, MA, USA) indiquent qu'un promoteur du gène de l'insuline possédant une séquence minisatellite VNTR courte, c'est-à-dire un allèle de lules pancréatiques $\beta$ qu'un promoteur ayant un VNTR long de classe III [3]. Pour obtenir ces résultats, les auteurs ont réalisé des constructions génétiques dans lesquelles un gène rapporteur est placé sous le contrôle du promoteur du gène de l'insuline en amont duquel sont positionnés, soit une séquence aléatoire, soit un VNTR de classe I, soit un VNTR de classe III. La construction est ensuite introduite par électroporation dans des îlots de Langerhans fotaux isolés de rats et l'activité du gène rapporteur est mesurée. La séquence VNTR a une action activatrice, d'autant plus marquée que le nombre de répétitions est grand. A l'inverse, Lucassen et al. d'Oxford (GB), et de l'hôpital Saint-Louis (Paris, France) montrent que des allèles de classe I semblent plus exprimés que des allèles de classe II. Ici, l'expérience consiste à transfecter dans des lignées établies de cellules $\beta$ tumorales un fragment d'ADN contenant le gène de l'insuline, ainsi que les régions bordantes en 5' et en 3' (préalablement défini comme le fragment de $4,1 \mathrm{~kb}$ ). L'accumulation de l'ARN d'insuline est appréciée par une expérience de protection d'une sonde ARN contre la dégradation par la RNase A [4]. La différence entre la stratégie expérimentale de l'équipe franco-anglaise de Lathrop [4] et de l'équipe américaine de German et Rutter [3] concerne les constructions génétiques, les cellules dans lesquelles elles sont introduites et le mode de dosage. Les Américains étudient simplement le pouvoir d'activation transcriptionnelle de la séquence minisa- tellite VNTR dans des cultures primaires d'îlots de Langerhans, alors que l'équipe européenne mesure l'accumulation de l'ARN messager insuline dans des cellules tumorales contenant la totalité du gène et des régions en amont et en aval. Dans ce dernier cas, les allèles de classe I et de classe III ne diffèrent pas seulement par le nombre de répétitions des VNTR, mais aussi par d'autres sites polymorphes situés en amont, à l'intérieur et en aval du gène. De plus, une augmentation de la quantité d'ARN messager produit par des allèles de classe I pourrait être due à une plus grande stabilité de ce messager et non pas à une augmentation de la transcription. Il est tout à fait impératif que des études complémentaires permettent de résoudre ces ambiguités.

De toutes façons, une modification du niveau d'expression du gène insuline n'explique pas aisément une susceptibilité particulière à un diabète de type I, c'est-à-dire à cette maladie auto-immune détruisant les cellules $\beta$ des îlots de Langerhans. Si la susceptibilité est associée à une augmentation de l'expression du gène de l'insuline, c'est-à-dire la synthèse de l'hormone, comme le suggèrent Lucassen et al. [4] et également Bennet el al. [2], peut-on considérer qu'une hyperproduction puisse être un facteur de rupture d'une tolérance immunologique? Ou encore qu'une synthèse accrue de cette hormone abondante dans les cellules $\beta$ puisse finir par endommager la cellule du fait d'une trop importante consommation d'énergie et d'une 
moindre résistance à des stress, oxydatifs, par exemple? Dans cette dernière hypothèse, ce seraient ces modifications cellulaires qui constitueraient le facteur déclenchant d'une réponse immune. Une autre hypothèse serait que ce que l'on appelle des allèles de classe 1 soient, en réalité, très hétérogènes, l'allèle utilisé par Kennedy el al. [3] n’étant justement pas particulièrement associé à une susceptibilité au diabète [2]. En conclusion, l'un des gènes importants de susceptibilité au diabète auto-immun de type I semble être le gène d'insuline lui-même, mais on ne sait pas encore si la susceptibilité est notée en cas d'augmentation ou de diminution de son expression. Nul doute qu'une réponse définitive à cette question devra être donnée dans les prochaines années et qu'elle permettra alors d'échafauder des mécanismes physiopathologiques d'apparition de la maladie autoimmune... qui resteront à tester.

A.K.

I. Khalil I, Lepage V. Degos I., (Galibert F, Deschamps 1, Hors f. Diabète insulinodépendant et HL A-1)Q: un modèle moléculaire de susceptibilité. médecine/sciences $1991 ; 7: 235-9$.

2. Bennet ST, Lucassen AM, (oough Sc.t, Powell E.E, Undlien DE, Pritchard L.E, Merriman ME, Kinwaguchi $Y$, Dronslield MJ, Pociot $F$. Nerup J, Bouzckri N, (ambon-Thomsen A, Ronnigen, kS, Barnetl AH, Bain SC; Todd JA. Susceptibilin to human type I diabetes at IDDDM2 is detcrmined by tandem repeat variation at the insulin gene minisatellite locus. Nature (jenet 1995; 9: 284-92.

3. Kennedy (;), (ierman MS, Rutter WJ. The minisatellite in the diabetes susceptibility locus IDI)M2 regulates insulin transcription. Nature (ienet $1995 ; 9$ : 293-8.

4. L.ucassen AM, Screaton (;R, Julicr (, Elliott T], lathrop $M$, Bell Jl. Regulation of insulin gene expression by the II)IDM associated, insulin locus haplotspe. Hum Mol (ienet 1995; 4 : 501-t).

\section{BRÈVES}

Le spermatozoïde anaconda. Comme pourrait le dire le poème enfantin de Desnos, un spermatozoïde humain de 35 mètres, ça n'existe pas, ça n'existe pas. Et pourtant, toutes proportions gardées, c'est bien des spermatozoïdes de ce degré de gigantisme qu' émettent les mâles Drosophila bifurca. Ces gamètes mesurent $58,3 \mathrm{~mm}$ et sont vingt fois plus longs que la mouche qui les fabrique... et 300 fois plus long que des spermatozoïdes humains! Chez ce mâle, le testicule de $67 \mathrm{~mm}$ de long représente près de $11 \%$ du poids sec de l'animal. Ces chiffres représentent un record méritant de figurer dans le livre Guinness; les précédents records étaient également tenus par des drosophiles, ce qui signifie que ce gigantisme des gamètes mâles a été conservé au cours de l'évolution sur plusieurs centaines de millions d'années. Cette situation contraste évidemment avec celle à laquelle nous sommes habitués, où les mâles usent avec prodigalité d'un nombre de gamètes de très petite taille excédant de loin leur espérance de reproduction. Une première conséquence est que la maturité des mâles, dans ces espèces, est tardive, puisqu'il leur faut le temps de fabriquer ces gonades et ces spermatozoïdes immenses (la maturité sexuelle de I). bifurca est à sept jours chez les femelles et dix-sept jours chez les mâles). Quel avantage sélectif a bien pu conserver une telle bizarrerie au cours de l'évolution des drosophiles? Peut-être les spermatozoïdes géants et leur immense flagelle sont-ils particulièrement efficaces pour féconder les ovocytes? Une autre hypothèse serait que, contrairement à ce qui se passe dans l'immense majorité des autres espèces, le spermatozoïde contribue aux fonctions du zygote postérieurement à la fécondation, lui apportant des protéines, des messagers et des organites cytoplasmiques. Par exemple, dans ces espèces, l'hérédité mitochondriale cesse évidemment d'être exclusive- ment maternelle. Cependant, un vingtième seulement du spermatozoïde géant de I). bifurca pénètre dans l'ovocyte, suggérant que la contribution à la constitution du zygote pourrait n'être pas considérable. Ainsi, l'avantage qu'ont les mouches mâles à fabriquer de tels spermatozoïdes anaconda reste énigmatique [1].

[1. Pitnick S, et al. Nature 1995; 375 : 109.]

U Un nouveau récepteur de bactéries sur les macrophages. Les macrophages ont un pouvoir très développé de reconnaissance, d'internalisation et de destruction. Les mécanismes de reconnaissance de protéines et de micro-organismes par les macrophages sont mal connus et parfois liés à l'expression de familles de récepteurs spécifiques de certaines caractéristiques structurales des agents étrangers. La famille des récepteurs scavenger des macrophages joue à ce titre un rôle fondamental dans la défense de l'organisme. Un nouveau type de récepteur (MARCO), ayant des ressemblances structurales avec le collagène et les récepteurs scavenger de type 1 , vient d'être identifié [1]. Il est exprimé par les macrophages de la rate et des ganglions et non par les macrophages du foie et des poumons comme les récepteurs scavenger. Il se lie aux bactéries mais pas aux levures. Il est aussi capable de fixer les LDL (low density lipoproteins) acétylées, ce qui suggère qu'il participe à la clairance de certains composants sériques.

[1. Elomaa (), et al. Cell $1995 ; 80$ : 603-9.]
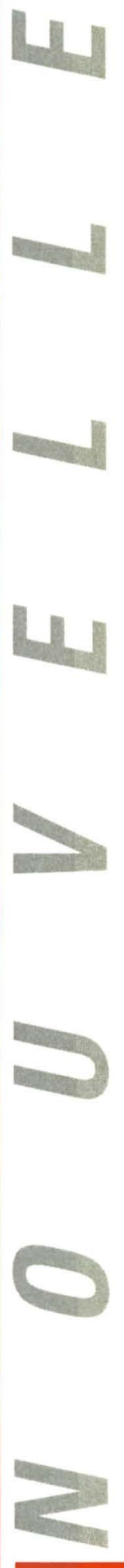